\title{
New matrix-free reference material for ethene in the form of optical fibres
}

\author{
Marta Słomińska • Piotr Konieczka • Jacek Namieśnik
}

Received: 27 August 2012 /Revised: 8 November 2012 / Accepted: 13 November 2012 /Published online: 14 December 2012

(C) The Author(s) 2012. This article is published with open access at Springerlink.com

\begin{abstract}
Reference materials are indispensable in the quality control and quality assurance of analytical measurements. One novel approach to the generation of standard gaseous mixtures of toxic, reactive, volatile, labile, and malodorous substances involves thermal decomposition or rearrangement, under defined temperature conditions, of compounds immobilized, by chemical bonding, on the surface of an appropriate carrier to release specific amounts of a volatile compound. In this technique the type of support used to immobilize the compound is extremely important, because the amount of analyte released depends directly on the choice of material. In this paper we report the novel preparation of a matrix-free ethene standard in the form of glass fibres coated with a thin layer of aluminium, the surface of which is modified by reaction with a specific compound. As a result of thermal decomposition of this compound, gaseous ethene is formed. In this paper we present the results obtained from tests of stability and homogeneity, two properties of crucial importance in the preparation of reference materials, by comparison of a series of results obtained for randomly chosen samples of the reference material. Interlaboratory comparative studies resulted in determination of a reference value for the ethene formed after thermal decomposition of the surface compound ((2.12 \pm 0.14$)$ ng per fibre).
\end{abstract}

Keywords Air - Gases · Reference materials · Quality assurance $\cdot$ Control

\section{Introduction}

Use of standards with matrix compositions and analyte concentrations resembling those of environmental samples

M. Słomińska $(\bowtie) \cdot$ P. Konieczka $\cdot$ J. Namieśnik

Chemical Faculty, Department of Analytical Chemistry,

Gdansk University of Technology, Narutowicza 11/12 Str,

80-233 Gdansk, Poland

e-mail: korpala.m@poczta.onet.pl enhances the reliability of analytical results [1-7]. Preparation of standard gas mixtures is much more difficult than the manufacture of liquid and solid reference materials (because of the character of many gaseous compounds - toxic, reactive, volatile, and unstable).

Methodological approaches for producing matrix-free standards of gas mixtures have advanced rapidly in recent years. Among static and dynamic techniques available for the preparation of standard gas mixtures, thermal decomposition of a surface compound has recently become popular [8-11].

At the Department of Analytical Chemistry of Gdansk University of Technology, research on the application of thermal-decomposition techniques has been conducted for 15 years. These studies have been focused on:

1. chemical modification of appropriate solid materials to obtain the desired surface compounds;

2. determination of optimum conditions for thermal decomposition of the surface compounds to obtain welldefined quantities of the desired analytes; and

3. development of quick and convenient calibration techniques.

The first trials on thermal decomposition were performed with silica gel as the carrier. Better carriers are now being sought to reduce the laborious and time-consuming modification of carrier surfaces, to eliminate errors that arise during weighing of the silica gel, and to eliminate adsorption of contaminants by the surface [11-13].

The carrier of a surface compound is crucially important during thermal decomposition of adsorbed analytes, because it directly affects the amount of the standard released and the repeatability of the results. Use of glass fibres as a carrier for the surface compound is an innovative means of production of matrix-free gaseous reference materials.

The purpose of this research was to produce a novel matrix-free ethene standard by thermal decomposition of a 
surface compound. The starting materials for the desired standard were samples of glass fibre $3 \mathrm{~cm}$ long, to give the fibre greater mechanical strength. Ethene is formed as a result of thermal decomposition of a compound chemically bound to the carrier surface.

\section{Experimental}

Chemical modification of glass fibres

Glass fibres (Cezar International Group, Photonics Technology) were $3 \mathrm{~cm}$ long coated with a thin layer of aluminium (660/680/860 $\mu \mathrm{m}$ external diameter of quartz/ external diameter of germanium-doped quartz/external diameter of aluminium).

The fibres were surface-modified with a compound which released ethene during thermal decomposition.

To modify the surface of glass fibres with an appropriate surface compound, several reactions were investigated. Modification was performed at the Department of Chemical Technology by Professor J.F. Biernat's research group. The glass fibres were dried at $120{ }^{\circ} \mathrm{C}$ for $8 \mathrm{~h}$ to remove all adsorbed water. They were then placed in a flask containing $60 \mathrm{~mL}$ toluene and $0.025 \mathrm{~mol}$ (3-chloropropyl)trimethoxysilane. Reaction was performed at $80{ }^{\circ} \mathrm{C}$ for $6 \mathrm{~h}$. The silanized carrier was isolated by filtration, washed successively with toluene, methanol, and acetone, and finally dried under vacuum. The product was immersed in toluene $(60 \mathrm{~mL})$ containing $0.025 \mathrm{~mol}$ diethylamine. The solution was then heated at $80{ }^{\circ} \mathrm{C}$ for $6 \mathrm{~h}$. After completion of the reaction, the product was again isolated by filtration, washed with water, and vacuum-dried. In the final stage of the synthesis $10 \mathrm{~mL} 30 \% \mathrm{H}_{2} \mathrm{O}_{2}$ was added. The modified carrier thereby obtained was carefully washed and dried [13].

The research material in the form of samples of glass fibre $3 \mathrm{~cm}$ long, modified with a surface compound that would release ethene on thermal decomposition, were divided into single packages. A single package was a glass bottle containing five surface-modified glass fibre samples selected at random from 1,500 such fibres. In this way 300 bottles of this new "candidate" for a reference material were prepared.

\section{Chromatographic conditions}

Homogeneity and stability measurements were performed by gas chromatography with a Hewlett-Packard Series II 5890 instrument with a flame ionization detector (FID) and fitted with a $100 \times 0.3 \mathrm{~cm}$ Porapak Q column $(80-100$ mesh). Isothermal chromatography was performed at $40^{\circ}$ C. The carrier gas was helium (flow rate $12 \mathrm{~mL} \mathrm{~min}^{-1}$ ).

\section{Calibration}

The GC system was calibrated by use of a standard gas mixture containing a strictly defined concentration of ethene $(19.8 \pm 0.4 \mathrm{ppm})$ in nitrogen, obtained from Linde Gas.

The linear range of the calibration curve was from 1.2 to $3.8 \mathrm{ng}$ ethene. The equation for the calibration curve was

$y=2364.4 x+260\left(y=b x+a ; r=0.999 ; S_{a}=115 ; S_{b}=42\right)$

where

$y \quad$ is the FID response expressed as peak area (conventional units)

$x$ is the mass of ethene (ng), and

$r$ is the correlation coefficient.

\section{Results}

Determination of homogeneity and stability

Determination of homogeneity was performed on the contents of seven different bottles. The samples for homogeneity testing were selected at random. Three fibres from each bottle were used for the tests (one bottle contains five 3-cmlong fibres).

The homogeneity tests were performed by thermal decomposition of the surface compound coupled with GCFID. A portable thermal desorber was used for generating, and dosing with, the volatile analyte (ethene). Both withinbottle and between-bottle homogeneity were tested.

Within and between-bottle homogeneity

Within-bottle homogeneity was tested by performing measurements on three modified fibres randomly selected from a bottle [2]. The gas mixture generated was analysed by GCFID. On the basis of the results obtained for three fibres from one bottle, within-bottle homogeneity was determined. For individual bottles the mean value and uncertainty were calculated. Mean values obtained for individual bottles were used for calculation of between-bottle mean value and uncertainty.

Figure 1 shows the results obtained from the homogeneity tests.

The average mass of ethene released from a single fibre was calculated from the results of tests performed for seven different bottles of material:

$$
\begin{aligned}
& m_{\text {ethene }}=\bar{X} \pm U(k=2) \\
& m_{\text {ethene }}=2.151 \pm 0.029[n g] \\
& u_{r(b b)}=\frac{0,0143}{2,151} \cdot 100 \%=0.66 \%
\end{aligned}
$$




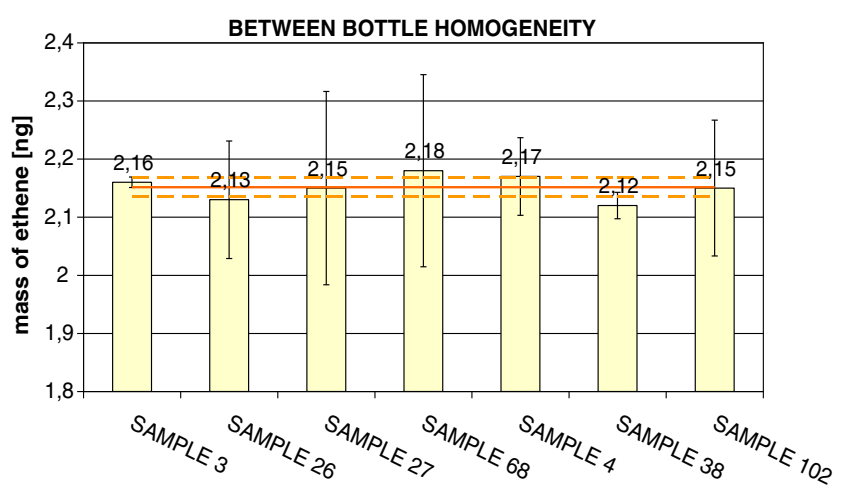

Fig. 1 Results obtained from measurements using TD-GC-FID

where

$X_{\text {avg }}$ is the mean of the mean values obtained for the seven individual bottles

$U \quad$ is the expanded uncertainty because of the homogeneity of the material, and

$u_{\mathrm{r}(\mathrm{bb})}$ is the relative standard uncertainty because of the homogeneity of the material.

Quantitative determination of the ethene indicated the material in each bottle was homogeneous. It was established that a single glass fibre coated with a suitable surface compound could be used as a matrix-free reference material for ethene.

\section{Determination of stability}

The fibres were prepared in October 2009. Stability tests were performed over a period of 12 months at six-month intervals. The stability tests started in March 2011 after storage of the fibres for 18 months.
Each determination of stability was performed for two different bottles stored at room temperature and two different bottles stored under refrigeration $\left(-18{ }^{\circ} \mathrm{C}\right.$ as reference conditions). Thus twelve bottles were used in the stability tests.

The tests were performed by the same procedure as was used for determining homogeneity [2]. The mass of ethene generated by thermal decomposition was determined both for samples stored at room temperature and for samples stored at $-18^{\circ} \mathrm{C}$.

$$
\begin{aligned}
& m_{\text {ethene }}=\bar{X} \pm U(k=2) \\
& m_{\text {ethene }}=2.101 \pm 0.080[n g] \\
& u_{r(\text { stab })}=\frac{0,040}{2,101} \cdot 100 \%=1.9 \%
\end{aligned}
$$

where

$X_{\text {avg }}$ is the mean of the mean values obtained for the 12 individual bottles

$U \quad$ is the value of the expanded uncertainty because of the stability of the material, and

$u_{\mathrm{r}(\mathrm{stab})} \quad$ is the relative standard uncertainty because of the stability of the material.

The results are shown in Fig. 2. The results from the long-term stability tests showed the material was stable whether stored at room temperature or under refrigeration. No changes in the amounts of ethene liberated were recorded during the storage period tested (30 months from the moment the fibres were chemically modified).

Statistical evaluation of the stability and homogeneity of the material

Homogeneity and stability are directly dependent on the mass of ethene formed by thermal decomposition of the surface compound. Statistical evaluation of homogeneity
Fig. 2 Comparison of the longterm stability of fibres stored at room temperature and at reduced temperature for 12 months
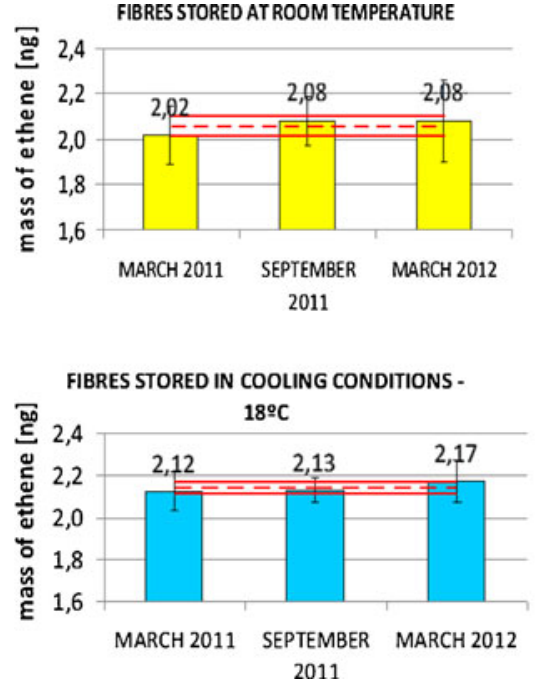
Table 1 Statistical evaluation of the material's homogeneity and stability

\begin{tabular}{lll}
\hline$Y=b X+a$ & Homogeneity & Stability \\
\hline Average mass of ethene (ng per fibre) & $2.151 \pm 0.029$ & $2.100 \pm 0.080$ \\
Standard deviation, $S$ & 0.021 & 0.056 \\
Intercept, $a$ & 2.156 & 1.977 \\
Standard deviation, $S_{\mathrm{a}}$ & 0.019 & 0.12 \\
Calculated Student's $t$ value, $t_{\text {calc }}$ & 0.032 & 1.3 \\
Slope of the straight line, $b$ & -0.0011 & 0.0050 \\
Standard deviation, $S_{\mathrm{b}}$ & 0.0044 & 0.0047 \\
Calculated Student's $t$ value & 0.25 & 1.064 \\
Critical Student's value, $t_{\text {crit }}$ & $2.571(f=n-2=5, \alpha=0.05)$ & $2.77(f=n-2=4, \alpha=0.05)$ \\
Correlation coefficient, $r_{\text {calc }}$ & -0.11 & 0.46 \\
Critical $r$ value, $r_{\text {crit }}$ & $0.75(f=n-2=5, \alpha=0.05)$ & $0.81(f=n-2=4, \alpha=0.05)$ \\
\hline
\end{tabular}

and stability was performed on the basis of the correlation coefficient $(r)$, the slope $(b)$, and the intercept $(a)$ (Table 1).

On the basis of the calculated value of the correlation coefficient there was no correlation between the mass of ethene formed in the thermal decomposition process and temperature or time of storage (the calculated value was compared with the critical value $\left.r_{\mathrm{kr}}>r_{\mathrm{cal}}\right)$.

Theoretically the values of the regression line should be $a=X_{\text {avg }}$ and $b=0$.

The intercept $(a)$ was not significantly different from the experimentally determined mass of ethene both in the homogeneity test $\left(t_{\text {crit }}(5 ; 0.05)=2.57>t_{\text {calc }}=0.032\right)$ and in the stability test $\left(t_{\text {crit }}(4 ; 0.05)=2.77>t_{\text {calc }}=1.3\right)$.

The slope of the line $(b)$ was not significantly different from 0 both in the homogeneity test $\left(t_{\text {crit }}(5 ; 0.05)=2.57>t_{\text {calc }}=0.25\right)$ and in the stability test $\left(t_{\text {crit }}(4 ; 0.05)=2.77>t_{\text {calc }}=1.064\right)$.

Interlaboratory comparison-certification campaign

Three research groups from the Chemical Faculty, Gdansk University of Technology, participated in the interlaboratory comparisons. Each laboratory received three bottles of test material. No particular measurement technique was imposed, nor were any measurement conditions stipulated for quantitative determination of the ethene formed by thermal decomposition of the surface compound on the glass fibre carrier. All three groups used TD-GC-FID for their determinations. The chromatographic conditions in the different laboratories are listed in Table 2.

The results obtained during the interlaboratory comparison are shown in Fig. 3.

$$
\begin{aligned}
& m_{\text {ethene }}=\bar{X} \pm U(k=2) \\
& m_{\text {ethene }}=2.12 \pm 0.11[n g] \\
& u_{r(\text { cert })}=\frac{0,055}{2,124} \cdot 100 \%=2.6 \%
\end{aligned}
$$

where

$X_{\text {avg }} \quad$ is the mean value obtained during the interlaboratory comparison for 16 bottles of material

$U \quad$ is the value of the expanded uncertainty in the interlaboratory comparison, and

$u_{\mathrm{r} \text { (cert) }} \quad$ is the relative standard uncertainty in the certification process.

Determination of certified value and estimate of uncertainty

The average mass of ethene released during thermal

\begin{tabular}{|c|c|c|c|c|c|c|}
\hline $\begin{array}{l}\text { Laboratory } \\
\text { code }\end{array}$ & Chromatograph & $\begin{array}{l}\text { Method of } \\
\text { calibration }\end{array}$ & $\begin{array}{l}\text { Flow rate } \\
\left(\mathrm{mL} \min ^{-1}\right)\end{array}$ & $\begin{array}{l}\text { Oven temperature } \\
\left({ }^{\circ} \mathrm{C}\right)\end{array}$ & $\begin{array}{l}\text { Carrier } \\
\text { gas }\end{array}$ & Column \\
\hline LAB 1 & $\begin{array}{l}\text { Perkin-Elmer } \\
\text { Clarus } 500\end{array}$ & External standard & 17 & 105 & Nitrogen & $\begin{array}{l}\text { Porapak Q ( } 80-100 \text { mesh), column length } \\
100 \mathrm{~cm} \text {, column diameter } 0.3 \mathrm{~cm}\end{array}$ \\
\hline LAB 2 & $\begin{array}{l}\text { Hewlett-Packard } \\
\text { Series II } 5890\end{array}$ & External standard & 20 & 35 & Helium & $\begin{array}{l}20 \% \text { squalane on Chromosorb P }(100-120 \\
\text { mesh), diameter } 1 / 8 \text { inch, length } 95 \mathrm{~cm}\end{array}$ \\
\hline LAB 3 & $\begin{array}{l}\text { Hewlett-Packard } \\
\text { Series II } 5890\end{array}$ & External standard & 12 & 40 & Helium & $\begin{array}{l}\text { Carboxen } 1010 \text { PLOT capillary column } \\
(30 \mathrm{~m} \times 0.53 \mathrm{~mm})\end{array}$ \\
\hline
\end{tabular}
decomposition of the surface compound on the fibre

Table 2 Chromatographic conditions used for determination of ethene in the laboratories participating in the interlaboratory comparison 
Fig. 3 Results from the interlaboratory comparison

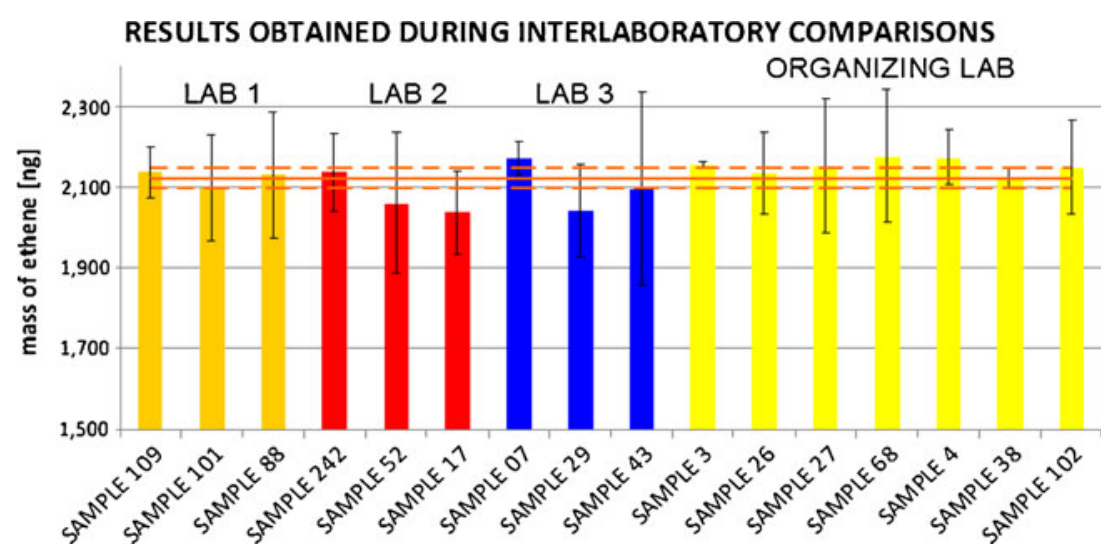

surface was calculated on the basis of the results obtained in all the laboratories which participated in the interlaboratory comparison. The uncertainty because of the inhomogeneity of the material was calculated from the standard deviations for the particular bottles (Fig. 1) [2, 4].

The uncertainty in the stability of the material was calculated from the standard deviations of the results obtained for samples under reference conditions and for samples stored at room temperature (Fig. 2) [2, 4].

The reference value for ethene was calculated as the arithmetic mean for the 16 bottles of material. First, the mean value was calculated for the individual bottle (three of the five fibres), then the mean value of the means was calculated (Fig. 3).

The complex expanded uncertainty of the reference value (certified value) was calculated taking into account all the sources of uncertainty affecting the reference value, in accordance with the relationship (Fig. 4) [2, 4]:

$$
U_{C R M}=k \sqrt{u_{c e r t}^{2}+u_{b b}^{2}+u_{\text {stab }}^{2}}
$$
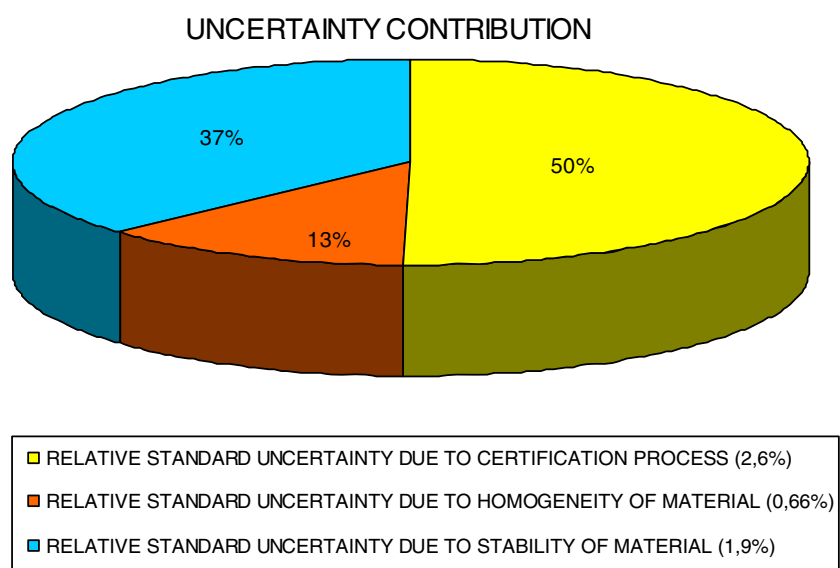

Fig. 4 Contributions to the expanded uncertainty value where $U_{\mathrm{CRM}}=0.14 \mathrm{ng}$ per fibre, $u_{\text {cert }}$ is the standard uncertainty because of the certification process for the material, $u_{\mathrm{bb}}$ is the standard uncertainty because of homogeneity, $u_{\text {stab }}$ is the standard uncertainty because of stability, and $k,=2$, is the coverage factor for $95 \%$ confidence interval.

On the basis of the results obtained during the interlaboratory comparison the reference value and the expanded uncertainty for ethene released from the glass fibre were calculated as $2.12 \pm 0.14 \mathrm{ng}$ per fibre.

\section{Conclusion}

The ethene standard produced in the form of glass fibres coated with a thin layer of aluminium chemically modified with specific compound which produces ethene on thermal decomposition can be regarded as a reference material. This research has led to innovative preparation of a gaseous reference material and the possibility of investigation of the applicability of appropriate fibres for generating standard mixtures of volatile organic compounds by thermal decomposition of immobilized compounds.

These results indicate it is possible to:

- reduce analysis time;

- eliminate sources of error; and

- achieve more precise and accurate analysis.

Moreover, this form of reference material is a useful tool for routine screening in daily laboratory practice.

Acknowledgments This research was partially funded by the Polish Ministry of Science and Higher Education (KBN NN204 264137). Further funding was received from the grant programme MISTRZ (2009 edition). Marta Słomińska expresses her gratitude for financial support from the funds of the project "Development of interdisciplinary postgraduate studies in modern technologies at the Gdansk University of Technology" (Project: POKL.04.01.01-00-368/09). 
Open Access This article is distributed under the terms of the Creative Commons Attribution License which permits any use, distribution, and reproduction in any medium, provided the original author(s) and the source are credited.

\section{References}

1. International Standard Organization, ISO GUIDE 30 (1992) Trends and Definitions used in Connection with Reference Materials, ISO. Geneva, Switzerland

2. International Standard Organization, ISO GUIDE 35 (2006) References materials - general and statistical principles for certification, ISO. Geneva, Switzerland

3. Konieczka P, Namieśnik J (2009) Quality assurance and quality control in the analytical chemical laboratory, a practical approach. CRC Press
4. Konieczka P, Namieśnik J (2007) Ocena i kontrola jakości wyników pomiarów analitycznych. WNT, Warszawa

5. Koziel JA, Martos PA, Pawliszyn J (2004) J Chromatogr A 1025:3-9

6. Hafkenscheid T, Baldan A, Quincey P, Cortez L, Dias F, Brown R, Wright M, Gerboles M, Saunders K (2005) Atmos Environ 39:3947-3965

7. Rhoderick GC (2003) J Chromatogr A 1017:131-139

8. Quevauviller P, Donard OFX (2001) Trends Anal Chem 20:600-613

9. Słomińska M, Konieczka P, Namieśnik J (2010) Trends Anal Chem 29:419-429

10. Przyk E, Konieczka P, Szczygielska-Tao J, Tischner R, Biernat JF, Namieśnik J (2001) J Chromatogr A 928:99-108

11. Świtaj-Zawadka A, Konieczka P, Szczygielska-Tao J (2004) J Chromatogr A 1033:145-151

12. Przyk E, Konieczka P, Szczygielska-Tao J, Biernat JF, Namieśnik J (2001) J Sep Sci 24:226-229

13. Kupiec K, Konarski P, Konieczka P, Namieśnik J (2009) Int J Mass Spectrom 286:11-16 